\title{
Activity Recognition In Smart Home Using Weighted Dempster- Shafer Theory
}

\author{
Elham Javadi ${ }^{1}$, Behzad Moshiri ${ }^{2}$ and Hadi Sadoghi Yazdi ${ }^{3}$ \\ ${ }^{1}$ Department of Computer Science and Research, \\ Islamic Azad University of Kerman, Iran \\ ${ }^{2}$ Control and Intelligent Processing Center of Excellence, \\ School of ECE, University of Tehran, Tehran, Iran \\ ${ }^{3}$ Department of Computer, Ferdowsi University of Mashhad, Mashhad, Iran \\ 1javadi.elh@gmail.com, ${ }^{2}$ moshiri@ut.ac.ir, ${ }^{3}$ h-sadoghi@um.ac.ir
}

\begin{abstract}
Smart homes are equipped with a variety of sensors to monitor the human activities. The information gathered from the heterogeneous sensors may not be always reliable and have different degrees of uncertainly. One of the most important techniques have been proposed to deal with uncertainty is Dempster-Shafer Theory (DST). In this paper, aims to define more precise sensor reliability and decrease uncertainty in sensor data in the activity recognition process within smart home. In the proposed method, in training step some models are built for per activity according extracted features from training samples and then in the prediction step when a new signal sensor is observed, the features extracted from that signal and applied to models and a weight is calculated for that sensor. These weights are considered as sensor reliability and uses in the decision making process.
\end{abstract}

Keywords: Uncertainty; Sensor reliability; Activity recognition; Dempster-shafer theory

\section{Introduction}

Advances in technology have provided the ability to equip home environment with different types of sensors, to provide improved living conditions and levels of independence for disabled and elderly people. One of the main supporting features of these homes is the ability to monitor the human activity. The information recorded from the sensors can be used to infer certain elements of health, well-being, and behavioral status [1, 2]. Considerable studies have been done toward the activity recognition by using a variety of different sensors such as audio, visual sensors and binary sensors. Simple, small and low cost binary sensors such as contact switches, temperature sensors, motion detectors unlike complex sensors such as cameras and microphones makes fewer interruptions for residents, and ensure privacy and comfort of the inhabitants in their homes.

The sensors may not always provide reliable information due to either faults, operational tolerance levels or corrupted data [3]. Thus, a data fusion technique is required to combine gathered data and manages uncertainty in sensor data to improve accuracies of results in the activity recognition process. Until now, many different methods such as based on fuzzy logic [4], neural networks [5], Bayesian network [6], hidden Markov models [7] are presented which may be used in the activity recognition within smart home. However, in these methods managing uncertainty does not consider as a key issue in the decision making process. Among data fusion techniques, Dempster-Shafer theory (DST) is an effective method that 
commonly used to manage uncertainty. Examples of applying the DST for activity recognition in smart homes have been previously reported in [3, 8, 9]. In [3] a general ontology generated for activities of daily living and used DST to infer about 'making drink'. In [10] a method for analyzing uncertainty and defining sensors reliability introduced.

In this paper aims to use historical data and activity patterns in order to assign a weight for each sensor to improve the reasoning performance. Section II represents DS theory and evidential operations. Section III represents details of proposed method for the toileting activity and finally in section IV simulations and conclusions are presented.

\section{Dempster-Shafer Theory}

DST is a mathematical theory of evidence. The seminal work on the subject is [11], which is an expansion of [12]. The core concepts in DST are the frame of discernment, mass functions and combination rule. The frame of discernment is used to present all possible states of a system, denoted by a set $\Omega$.The DS fusion process is based on $2^{\Omega}$ elements called propositions. Mass function represented by $\mathrm{m}$, defines a mapping of the power set to the interval $[0,1]$ with two conditions:

$$
\left\{\begin{array}{l}
m: 2^{\Omega} \rightarrow[0,1] \\
m(\phi)=0, \quad \sum m(A)=1 \\
A \subseteq \Omega
\end{array}\right.
$$

Which $2^{\Omega}$ is set of all subset of $\Omega$ and $\varphi$ is the empty set.

Belief function and plausibility function are the most important functions in evidence theory which represent lower and upper bounds of belief level of interest proposition.

$$
\begin{aligned}
& \operatorname{Bel}(A)=\sum_{B \subseteq A} m(B), \quad \forall A \subseteq \Omega \\
& \operatorname{Pls}(A)=1-\underset{B \cap A=\phi}{\sum_{B} m(B)}
\end{aligned}
$$

The interval between belief and plausibility values in a proposition represents the confidence and the width of the interval represents the amount of uncertainty.

For combining different pieces of evidence from various sources which have different levels of reliability, a discounting operator is required to represent levels of reliability of the evidence.

$$
m^{d}(A)= \begin{cases}(1-r) m(A), & A \subseteq \Theta \\ r+(1-r) m(\Theta), & A=\Theta\end{cases}
$$

Where $\mathrm{r}$

$\in[0,1]$ is the discou

while $r=1$ indicates the source is totally unreliable. For combining two pieces of evidence Dempster's rule of combination is used.

$$
\begin{gathered}
m_{1} \oplus m_{2}(A)= \\
\left\{\begin{aligned}
(1-k)^{-1} & \sum_{B \cap C=A} m_{1}(B) * m_{2}(C), \text { if } B \cap C \neq A \\
0 & \text { if } B \cap C=A
\end{aligned}\right.
\end{gathered}
$$


Which $k=\sum_{B \cap C \neq \Phi} m_{1}(B) * m_{2}(C)$ is interpreted as a measure of conflict among the various sources [13]. More than two piece of evidence, can be combined by Dempster's rule of combination.

$$
m 1 \oplus m 2 \oplus \ldots \oplus \ldots=(((m 1 \oplus m 2) \oplus \ldots) \oplus \ldots)
$$

Within smart homes, some activities have alternative sub-activities, then a maximum operation can be used to calculate the amount of belief and plausibility.

$$
\begin{aligned}
& \operatorname{Bel}(A)=\max (\operatorname{Bel}(B), \operatorname{Bel}(C)) \\
& \operatorname{Pls}(A)=\max (\operatorname{Pls}(B), \operatorname{Pls}(C))
\end{aligned}
$$

\section{Proposed Method}

In this paper, a weight is considered for each sensor which represents the similarity between signal sensor and signals in the training phase. As shown in Figure 1, in the training stage, using a feature window with length $L$ and extracts features in each window. The feature window is positioned at the first of each activity label and for next time is shifted $\Delta t$. Using the extracted features, 10 models are built for each sensor. In the testing stage, similar to training step a feature window is positioned at time $t$ and features are calculated. These features are applied to models and a weight for each sensor is obtained. the proposed method is named "weighted DST" (WDST). More details of proposed method will describe in the following.

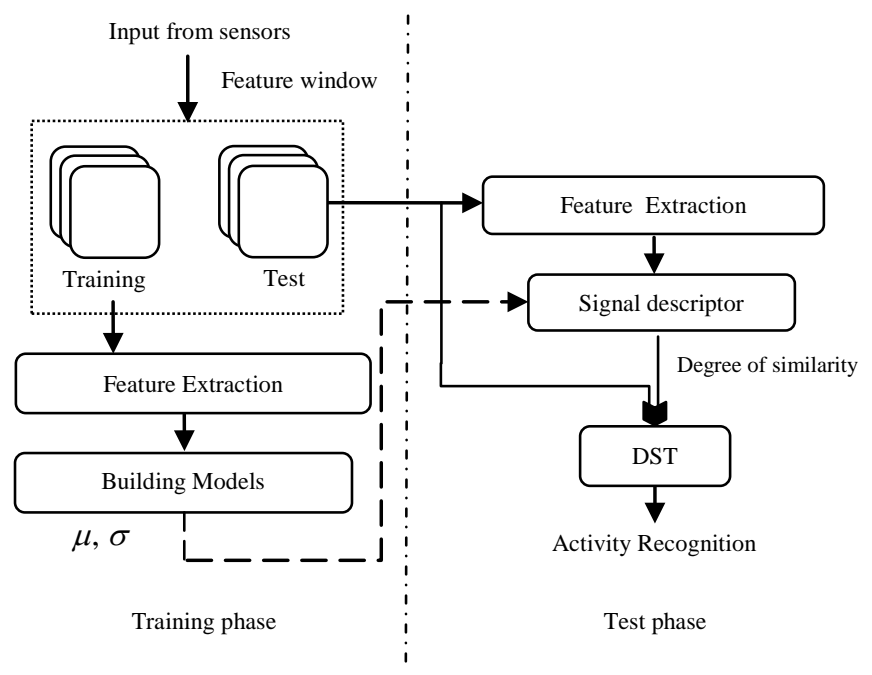

Figure 1. The Overall Structure of the Proposed Method

A) Generating training samples: In the training stage, training examples of each activity include all the activities which have similar labels in the dataset. For example, training samples of activity A includes all the activities with label A in the dataset as Figure 2. The other activities are labeled as "unknown activity". According to Figure 2, activity A has occurred for 3 times, so there are 3 training samples for the sensors that inhabitant used them during the activity A. Figure 3 is shown training samples for S1. 


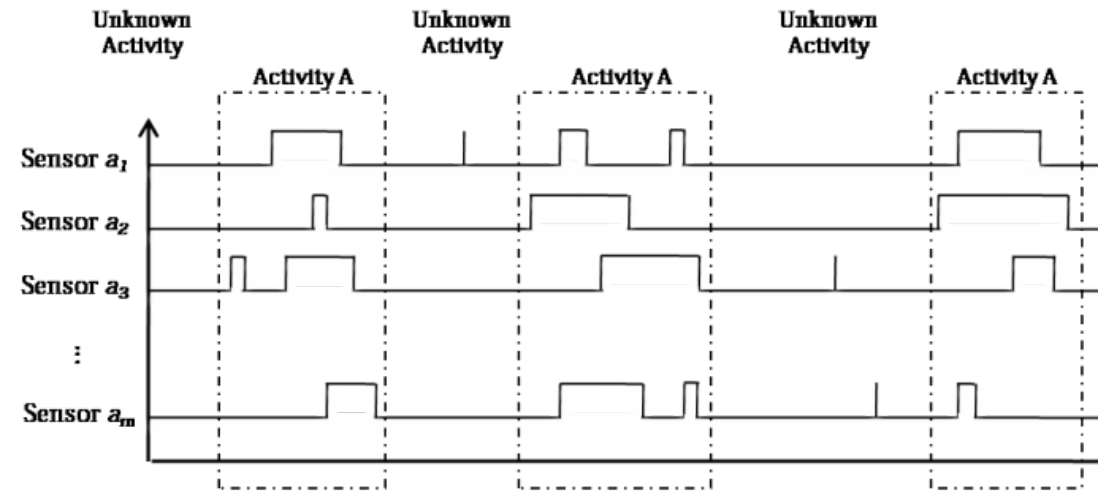

Figure 2. An Example of Different Occurrences of Activity A

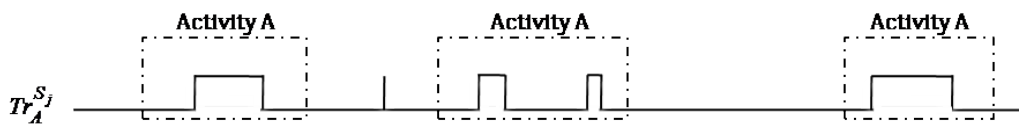

Figure 3. Training Samples for $\mathrm{S}_{1}$

Different activities have different mean lengths of time. Here, a feature window is used to extract features, and the length of the window is corresponded to mean of the activity length as carried out by the inhabitant within smart home. The feature window is positioned at the first of training sample and shift $\Delta t$ (which was half of the duration of the quickest activity) for next time [14].

B) Feature extraction: Features could be defined as primary and useful information on data collected by sensors. These features are considered as a feature vector. Here, using FFT[15] for feature extraction as represented in (9).

$$
F(u)=\sum_{x=0}^{L-1} f(x) e^{-j 2 \pi u x / L}, \quad u=0,1,2, \ldots, L-1
$$

For feature extraction, first training samples of each sensor is transformed to the frequency domain and then the first 10 Fourier coefficients are considered as primary features of that example. Feature matrix $\mathrm{F}$ is defined as:

$$
F=\left[\begin{array}{l}
\left(c_{1}: c_{10}\right)_{1}^{s_{1}},\left(c_{1}: c_{10}\right)_{1}^{s_{2}}, \cdots,\left(c_{1}: c_{10}\right)_{1}^{s_{m}} \\
\left(c_{1}: c_{10}\right)_{2}^{s_{1}},\left(c_{1}: c_{10}\right)_{2}^{s_{2}}, \cdots,\left(c_{1}: c_{10}\right)_{2}^{s_{m}} \\
\left(c_{1}: c_{10}\right)_{k}^{s_{1}},\left(c_{1}: c_{10}\right)_{k}^{s_{2}}, \cdots,\left(c_{1}: c_{10}\right)_{k}^{s_{m}}
\end{array}\right]
$$

Which $\left(c_{1}: c_{10}\right)_{j}^{S_{i}}$ represents the first 10 Fourier coefficients for sensor i in j'th times of occurring activity. $\mathrm{m}$ is the number of associated sensors which used during activity and $\mathrm{k}$ is the number of training samples.

C) Build models for each of sensors: According to the extracted feature from training phase, for each sensor 10 Gaussian probability density function corresponding to each set of 
10 Fourier coefficients are calculated. For example, from matrix F for k first coefficients (c1) for S1 has built a model and as for the rest. Finally for each sensor 10 models are built. Each density function is calculated using mean and variance of coefficients as shown Figure 4. In $F^{s_{j}}$ each row represents the 10 features for $s_{j}$ in each window and $F\left(u_{i}\right)_{l}^{s_{j}}$ is i'th Fourier coefficients for $l$ 'th sample of the sensor $s_{j}$.

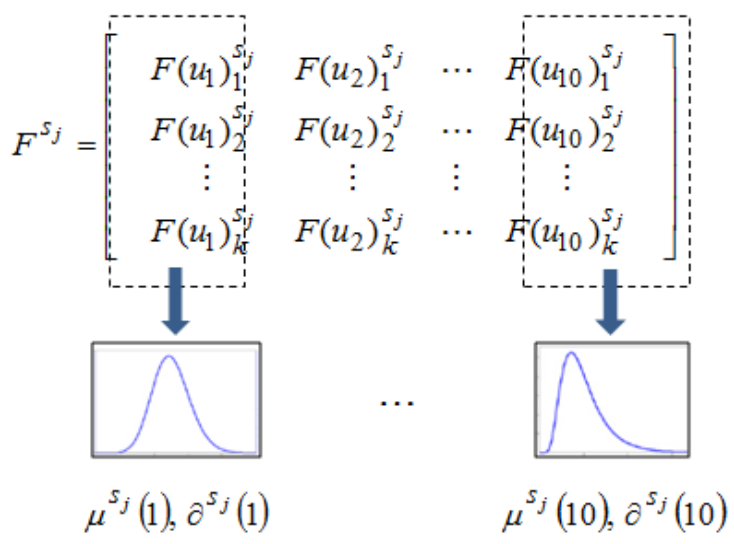

Figure 4. Building 10 Models for $S_{j}$

D) Testing phase (feature extraction and calculation degree of similarity): In this stage, according to Fig. 5 the feature window is positioned at the current time $t$ to analyze. The features are calculated from time t to time $t-L_{i}$ for each sensor and the feature window for the time is positioned at $t+\Delta t$. The features applied to models from training step and a weight is obtained for each sensor which represented similarity of that signal sensor to the training samples of the sensor.

$$
w_{i}=\sum_{j=1}^{10} d_{j}^{a} j, \quad i=1,2, \cdots, m
$$

which $d_{i}^{S_{i}}$ is membership degree of each signal sensor to its models. To calculate the weight of each sensor, the features applied to models and membership degree of each feature is calculated. Finally, the sensor's weight is calculated as (11).

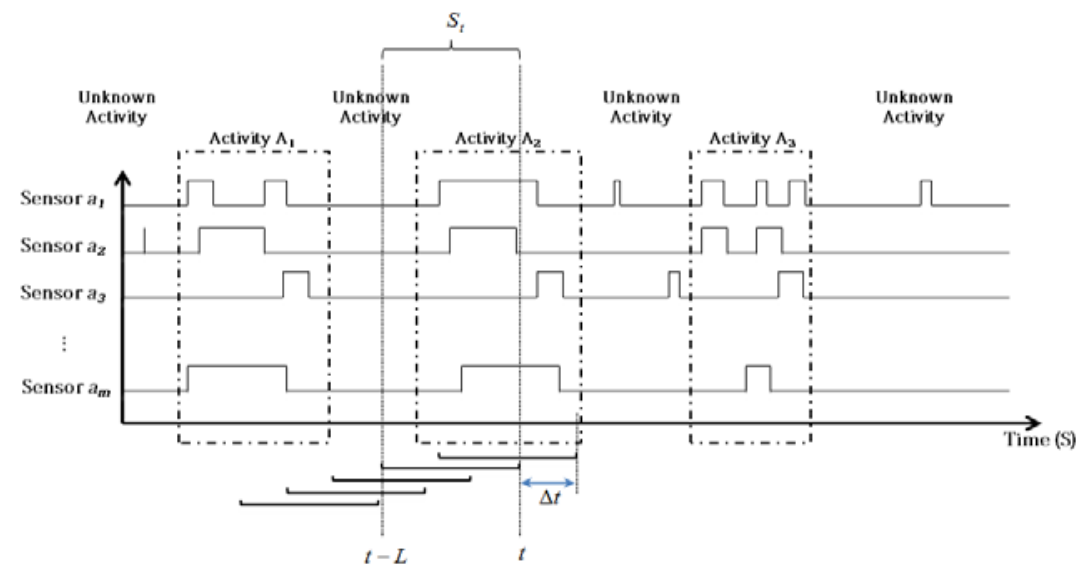

Figure 5. Moving Feature Window on Signal Sensors for Testing Phase 
E) Activity recognition Process: Activity recognition of daily living could be identified by the inhabitant's interaction with objects within smart home [14]. The associated sensors with attached on everyday objects can be used to detect interaction with objects and movement associated with activity. The interrelationships between the sensors and the activities can be represented by a hierarchical sketch [3]. In this paper, an ontology is considered for toileting activity and then used DST and calculated weights for inferring about the activity.

For example, consider the toileting activity. Consider the bathroom environment is equipped with 5 sensors is attached on 5 objects: light, Hot tap, cold tap, flush tank and bathroom cabinet. five contact sensors are attached to these objects from the database examined [14]: toilet light sensor (ID 101), bathroom hot tap sensor (ID 68), bathroom cold tap sensor (ID 88), bathroom cabinet sensor (ID 57), and flush sensor (ID 100).

In Figure 6 the ontology for toileting activity is shown. The information that could be used to characterize the activity of the inhabitant, including the room that the inhabitant is in, objects that the inhabitant interacts with, is referred as context [3]. In the Figure 6 the sensors, the object contexts and the activity are represented by circular, square and rectangular nodes respectively. The context is divided into two groups: core and accessory. Core contexts are the compulsory contexts, which must be interacted with when performing a certain activity. Accessory contexts could be considered as optional and may or may not be interacted within a specific activity. In the Figure 6 the sensors, the object contexts and the activity are represented by circle, square and rectangular nodes respectively. Any accessory contexts are connected to the activity node by the dash line. A diamond arrow with dash square represents contexts that are not directly associated with a sensor but rather deduced from a sensed context. Composite node is the composite of all core contexts related to the activity and shown as an eclipse node in the network [3].

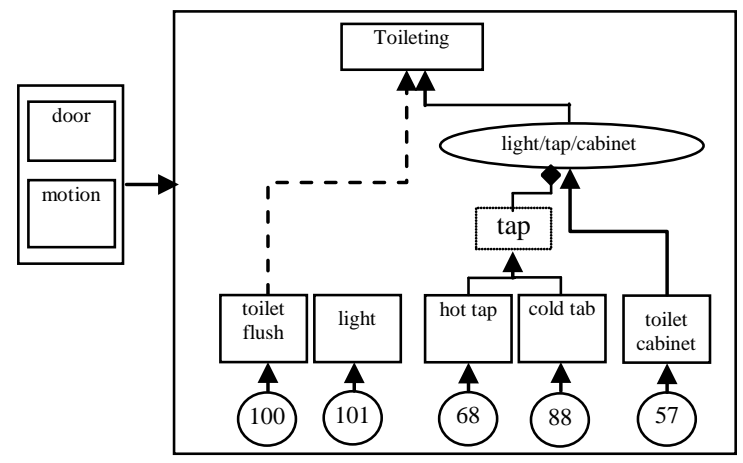

Figure 6. Ontology for Toileting Activity

To further explain the proposed method, a simple scenario is used [10]:

A man who walks into the bedroom. In the first instance the bedroom door sensor is triggered and the bathroom motion sensor is active. Then the system detects 3 sensors: bathroom light, flush and hot top becoming active. After about three minutes the door sensor and motion sensor are triggered again and turn inactive. During the 3 minute period the system didn't detect any other sensor activations. What really happened in the bathroom during this 3 minute period?

The bathroom door and motion sensors help to understand that activity happened in the bathroom. Within the bathroom, there are various activities related to these sensors such as "washing", "grooming" and "Toileting". 
In this paper aims to recognize toileting activity according to the ontology in Figure 6. First, the frame of discernment is defined for each node. Table I presents an example of the frame of discernment for each type of node.

\section{Table 1. Example of the Frame Of Discerment}

\begin{tabular}{|c|c|c|}
\hline name & Type & Frame of discernment \\
\hline slight & Sensor & $\{$ slight, $\neg$ slight $\}$ \\
\hline light & Context & $\{$ light, $\neg$ light $\}$ \\
\hline toileting & Activity & $\{$ toileting,, toileting $\}$ \\
\hline
\end{tabular}

The process of reasoning about the daily activities included the following steps:

1- Defining initial mass functions: After setting up the frame discernment, evidence on each sensor nodes are represented by mass functions.

$$
\begin{aligned}
& m_{\text {sflush }}(\{\text { sflush }\})=1, \\
& m_{\text {slight }}(\{\text { slight }\})=1, \\
& m_{\text {staphot }}(\{\text { staphot }\})=1, \\
& m_{\text {stapcold }}(\{\neg \text { stapcold }\})=1, \\
& m_{\text {scabinet }}(\{\neg \text { scabinet }\})=1
\end{aligned}
$$

2- Discounting sensor evidence: Sensors are working correctly at different rates: 0.95 for flush, light and cabinet sensors, 0.85 for hot tap and cold tap sensors. The discounted mass function can be calculated as follows:

$$
\begin{aligned}
& m_{\text {sflush }}^{r}(\{\text { sflush }\})=0.95, \\
& m_{\text {sflush }}^{r}(\{\text { sflush, } \neg \text { sflush }\})=0.05, \\
& m_{\text {slight }}^{r}(\{\text { slight }\})=0.95, \\
& m_{\text {slight }}^{r}(\{\text { slight }, \neg \text { slight }\})=0.05, \\
& m_{\text {staphot }}^{r}(\{\text { staphot }\})=0.85, \\
& m_{\text {staphot }}^{r}(\{\text { staphot }, \neg \text { staphot }\})=0.15, \\
& m_{\text {stapcold }}^{r}(\{\neg \text { stapcold }\})=0.85, \\
& m_{\text {stapcold }}^{r}(\{\text { stapcold }, \neg \text { stapcold }\})=0.15, \\
& m_{\text {scabinet }}^{r}(\{\neg \text { scabinet }\})=0.95, \\
& m_{\text {scabinet }}^{r}(\{\text { scabinet }, \neg \text { scabinet }\})=0.05
\end{aligned}
$$

3- Adding weights to mass functions: Consider weights $0.4,0.8,0.5,0.5$ and 0.75 for flush, light, hot tap, cold tap and cabinet sensors respectively. Mass functions are calculated as follows: 


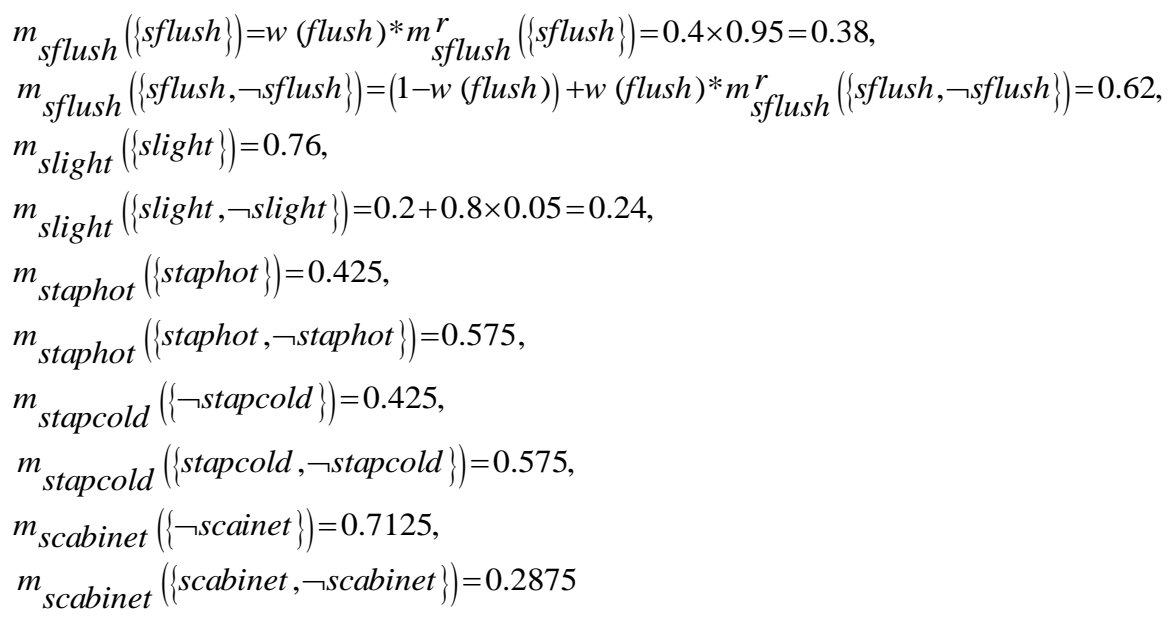

According to different weights of sensors, The mass functions are discounted because the observed signals are not completely similar to training samples.

4- Translating mass function to contexts: When a sensor activated, it means that inhabitant interacts with the associated context of that sensor. A sensor and its associated context have a compatibility relation which can be represented by a multivalued mapping. Therefore, mass function from sensor node can be translated to a context node by the multivalued mapping as follows:

$$
\begin{aligned}
& m_{\text {flush }}(\{\text { flush }\})=m_{\text {sflush }}(\{\text { sflush }\})=0.38, \\
& m_{\text {flush }}(\{\text { flush }, \neg \text { flush }\})=m_{\text {sflush }}(\{\text { sflush }, \neg \text { sflush }\})=0.62, \\
& m_{\text {light }}(\{\text { light }\})=m_{\text {slight }}(\{\text { slight }\})=0.76, \\
& m_{\text {light }}(\{\text { light }, \neg \text { light }\})=m_{\text {slight }}(\{\text { slight }, \neg \text { slight }\})=0.24, \\
& m_{\text {taphot }}(\{\text { taphot }\})=m_{\text {staphot }}(\{\text { staphot }\})=0.425, \\
& m_{\text {taphot }}(\{\text { taphot }, \neg \text { taphot }\})=m_{\text {staphot }}(\text { staphot }, \neg \text { staphot })=0.575, \\
& m_{\text {tapcold }}(\{\neg \text { tapcold }\})=m_{\text {stapcold }}(\{\neg \text { stapcold }\})=0.425, \\
& m_{\text {tapcold }}(\{\text { tapcold }, \neg \text { tapcold }\})=m_{\text {tapcold }}(\{\text { stapcold }, \neg \text { stapcold }\})=0.575, \\
& m_{\text {cabinet }}(\{\neg \text { cainet }\})=m_{\text {scabinet }}(\{\neg \text { scainet }\})=0.7125, \\
& m_{\text {cabinet }}(\{\text { cabinet }, \neg \text { cabinet }\})=m_{\text {scabinet }}(\{\text { scabinet }, \neg \text { scabinet }\})=0.2875
\end{aligned}
$$

The context "taphot" and "tapcold" are two alternatives of context "tap". The mass function for tap is calculated by maximization operation as follows:

$$
\begin{aligned}
& m_{\text {tap }}(\{\text { tap }, \neg \text { tap }\})=\max \left(m_{\text {tapc }}(\{\text { tapc }, \neg \text { tap }\}), m_{\text {taph }}(\{\text { taph }, \neg \text { taph }\})\right)=0.575, \\
& m_{\text {tap }}(\{\operatorname{tap}\})=\max \left(m_{\text {taph }}(\{\text { taph }\}), m_{\text {tapc }}(\{\text { tapc }\})\right)=0.425
\end{aligned}
$$

5- Calculating mass function for composite node: In Figure 6 "light, tap, cabinet" node is a composite node of core context light, tap and cabinet. For calculating mass function of composite node, the equally weighted sum operator is used: 


$$
\begin{gathered}
m_{(\text {ligh } / \text { tap } / \text { cabinet })}(\{(\text { ligh / tap / cabinet })\})=\frac{1}{3}\left(m_{\text {light }}(\{\text { light }\})\right. \\
\left.+m_{\text {tap }}(\{\text { tap }\})+m_{\text {cabinet }}(\{\text { cabinet }\})\right)=\frac{1}{3}(0.76+0.425+0)=0.395 \\
m_{(\text {ligh } / \text { tap } / \text { cabinet })}(\{\neg(\text { ligh / tap / cabinet })\})=\frac{1}{3}\left(m_{\text {light }}(\{\neg \text { light }\})\right. \\
\left.+m_{\text {tap }}(\{\neg \text { tap }\})+m_{\text {cabinet }}(\{\neg \text { cabinet }\})\right)=\frac{1}{3}(0+0+0.7125)=0.2375 \\
m_{(\text {ligh } / \text { tap / cabinet })}(\{(\text { ligh / tap / cabinet }), \neg(\text { ligh / tap / cabinet })\})= \\
\frac{1}{3}\left(m_{\text {light }}(\{\text { light }, \neg \text { light }\})+m_{\text {tap }}(\{\text { tap }, \neg \text { tap }\})\right. \\
\left.+m_{\text {cabinet }}(\{\text { cabinet }, \neg \text { cabinet }\})\right)= \\
\frac{1}{3}(0.24+0.575+0.2875)=0.3675
\end{gathered}
$$

6- Combining mass function on activity node: Mass function $\mathrm{m} 1, \mathrm{~m} 2$ on activity node from two different independent evidence sources are combined by Dempster' combination rule to achieve consensus:

$$
\begin{aligned}
& m(\{\text { Toileting }\})=m_{1} \oplus m_{2}(\{\text { Toileting }\})=0.5877 \\
& m(\{\neg \text { Toileting }\})=m_{1} \oplus m_{2}(\{\neg \text { Toileting }\})=0.1619 \\
& m(\{\text { Toiletin, } \neg \text { Toileting }\})= \\
& \quad m_{1} \oplus m_{2}(\{\text { Toiletin }, \neg \text { Toileting }\})=0.2502
\end{aligned}
$$

As a consequence of the aforementioned, the belief value of the toileting activity is obtained:

$$
\begin{aligned}
& \operatorname{Bel}(\{\text { Toileting }\})=m(\{\text { Toileting }\})=0.5877 \\
& \operatorname{Bel}(\{\neg \text { Toileting }\})=m(\{\neg \text { Toileting }\})=0.1619
\end{aligned}
$$

Hence, "Toileting" has performed with a high degree of confidence.

\section{Simulation and results analyzing}

The Proposed method has applied on a dataset collected from MIT Laboratory [14]. In their experiment, 77 sensors were installed in single-person apartment to collect data about human activity for two weeks. These sensors were installed in everyday objects such as drawers, refrigerators, containers, etc., to record opening-closing events (activation deactivation events) as the subject carried out everyday activities [14]. Here, the first apartment is used to investigate the proposed method. The evaluation was carried out by the leave-one-out cross-validation, i.e., 13 days of data were used for training and one day of data was used for testing. The evaluated performance was measured by accuracy detection rate of activity.

$$
\text { Ac }=\frac{T_{\text {Toilet }}+T_{\neg \text { Toilet }}}{T_{\text {Toilet }}+F_{\text {Toilet }}+T_{\neg \text { Toilet }}+F_{\neg \text { Toilet }}}
$$


Which $T_{\text {Toilet }}$ is The number of windows that toileting activity have occurred and system is correctly recognized. $F_{\text {Toilet }}$ is The number of windows that toileting activity has occurred and the system is incorrectly recognized. $T_{\neg \text { Toilet }}$ is the number of windows that toileting has not occurred and the system is correctly recognized. $F_{\neg \text { Toilet }}$ is The number of windows that toileting activity has not occurred and the system is incorrectly recognized. Using the dataset, the results attained are presented in Table II. The results show that the proposed method is improved compared to the DST.

\section{Table 2. Accuracy Rate for Toileting Activity}

\begin{tabular}{|c|c|}
\hline Algorithm name & Accuracy \\
\hline DST & $71.28 \%$ \\
\hline WDST & $80.61 \%$ \\
\hline
\end{tabular}

\section{Conclusion}

In this paper, a new method introduced for activity recognition within the smart home which named WDST. In the training phase, features extracted for each feature window with length $\mathrm{L}$, which positioned at the first of activity label and shifted $\Delta t$ for the next time. In each feature window Fourier transform used to extract features. These features are first 10 Fourier coefficients and used to build models for each sensor. In the testing phase, each feature window positioned at the current time t to analyze. The features calculated from time $t-L_{i}$ to time $t$ for each sensor and next time $t+\Delta t$. The features applied to obtain models from training step. A weight calculated for each sensor, which represented similarity of signal sensor to training samples of the sensor. These weights used to determine more precisely the sensor reliability. Finally, the ontology and DST used for the decision making. The results have been based on an analysis of a component of the MIT dataset [14] and have shown the proposed method improved compare to DST.

\section{References}

[1] D. Cook and S. Das, "Smart Environments: Technology, Protocols and Applications (Wiley Series on Parallel and Distributed Computing): Wiley-Interscience, (2004).

[2] H. Wu, M. Siegel and S. Ablay, "Sensor fusion for context understanding”, IEEE Instrumentation and Measurement Technology Conference, vol. 1, (2002), pp. 13-17.

[3] X. Hong, C. Nugent, M. Mulvenna, S. McClean, B. Scotney and S. Devlin, "Evidential fusion of sensor data for activity recognition in smart homes”, Pervasive Mob. Comput., vol. 5, pp. 236-252, (2009).

[4] M. Marin-Perianu, C. Lombriser, O. Amft, P. Havinga, G. Tr\, \\#246, and ster, "Distributed Activity Recognition with Fuzzy-Enabled Wireless Sensor Networks", Proceedings of the 4th IEEE international conference on Distributed Computing in Sensor Systems Santorini Island, Greece: Springer-Verlag, (2008).

[5] J.-Y. Yang, J.-S. Wang and Y.-P. Chen, "Using acceleration measurements for activity recognition: An effective learning algorithm for constructing neural classifiers”, Pattern Recogn. Lett., vol. 29, (2008), pp. 2213-2220.

[6] W. Huadong, M. Siegel, R. Stiefelhagen and Y. Jie, "Sensor fusion using Dempster-Shafer theory [for context-aware HCI]”, Instrumentation and Measurement Technology Conference, IMTC/2002, Proceedings of the 19th IEEE, vol. 1, (2002), pp. 7-12.

[7] H. H. Bui, S. Venkatesh and G. West, "Policy recognition in the abstract hidden Markov model”, J. Artif. Int. Res., vol. 17, (2002), pp. 451-499.

[8] J. Liao, Y. Bi and C. Nugent, “Activity Recognition for Smart Homes Using Dempster-Shafer Theory of Evidence Based on a Revised Lattice Structure”, Proceedings of the 2010 Sixth International Conference on Intelligent Environments: IEEE Computer Society, (2002).

[9] S. Mckeever, J. Ye, L. Coyle, C. Bleakley and S. Dobson, “Activity recognition using temporal evidence theory”, J. Ambient Intell. Smart Environ., vol. 2, pp. 253-269. 
[10] J. Liao, Y. Bi and C. Nugent, "Using the Dempster Shafer Theory of Evidence with a Revised Lattice Structure for Activity Recognition”, Trans. Info. Tech. Biomed., vol. 15, (2012), pp. 74-82.

[11] P. Dempster and H. U. C. M. D. O. STATISTICS., "Upper and Lower Probabilities Induced by a Multivalued Mapping”, Defense Technical Information Center, (1966).

[12] G. Shafer, “A Mathematical Theory of Evidence”, Princeton University Press, (1976).

[13] K. Sentz, S. Ferson and S. N. Laboratories, "Combination of Evidence in Dempster-Shafer Theory", Sandia National Laboratories, (2002).

[14] E. M. Tapia, M. I. o. T. S. o. Architecture, P. P. i. M. Arts and Sciences, "Activity Recognition in the Home Setting Using Simple and Ubiquitous Sensors”, Massachusetts Institute of Technology, School of Architecture and Planning, Program in Media Arts and Sciences, (2003).

[15] P. Duhamel and M. Vetterli, "Fast Fourier transforms: a tutorial review and a state of the art”, Signal Process, vol. 19, (1990), pp. 259-299. 
International Journal of Smart Home Vol.7, No.6 (2013) 